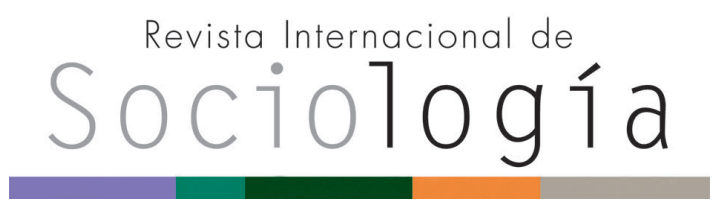

Revista Internacional de Sociología RIS

vol. 78 (3), e160, julio-septiembre, 2020, ISSN-L:0034-9712 https://doi.org/10.3989/ris.2020.78.3.19.083

\section{INMIGRACIÓN, LENGUA Y RENDIMIENTO ACADÉMICO EN ESPAÑA. UNA REVISIÓN SISTEMÁTICA DE LA LITERATURA}

\author{
Alberto Álvarez-Sotomayor \\ Universidad de Córdoba \\ aasotomayor@uco.es \\ ORCID iD: https://orcid.org/0000-0001-6174-1740
}

\author{
GLORIA MARTíNEZ-Cousinou \\ Universidad Loyola Andalucía \\ gmartinez@uloyola.es \\ ORCID iD: https://orcid.org/0000-0003-1971-6191
}

Cómo citar este artículo / Citation: Álvarez-Sotomayor, A. y G. Martínez-Cousinou. 2020. "Inmigración, lengua y rendimiento académico en España. Una revisión sistemática de la literatura". Revista Internacional de Sociología 78(3):e160. https://doi.org/10.3989/ris.2020.78.3.19.083

\begin{abstract}
RESUMEN
Desde los años noventa, han proliferado en España los trabajos que abordan las relaciones entre inmigración, dominio de la lengua escolar y rendimiento académico del alumnado con padres inmigrantes. En su mayoría defienden la hipótesis de la desventaja lingüística como clave para explicar su inferior rendimiento académico. Este artículo revisa esta literatura en el marco español, valorando sus contribuciones, limitaciones y lagunas. Pese a los avances, se concluye que la práctica ausencia de estudios que midan simultáneamente el dominio de la lengua escolar y el rendimiento académico imposibilita un conocimiento empírico riguroso de tal relación y, con ello, el contraste de dicha hipótesis. El tamaño de las muestras y de las submuestras de los grupos de origen y el adecuado control de determinadas variables relevantes son señalados como otros obstáculos a salvar en futuras investigaciones.
\end{abstract}

\section{Palabras Clave}

Dominio del español; Hijos de inmigrantes; Integración lingüística; Rendimiento escolar.

\section{IMMIGRATION, LANGUAGE AND ACADEMIC ACHIEVEMENT IN SPAIN. A SYSTEMATIC REVIEW}

Copyright: @ 2020 CSIC. Este es un artículo de acceso abierto distribuido bajo los términos de la licencia de uso y distribución Creative Commons Reconocimiento 4.0 Internacional (CC BY 4.0).

Recibido: 01/07/2019. Aceptado: 20/01/2020. Publicado online: 28/09/2020.

\section{ABstract}

Since the nineties, publications that deal with the relationships between immigration, proficiency in the language of instruction and academic performance of children of immigrants have multiplied in Spain. The majority defend the linguistic disadvantage hypothesis when explaining the poorer performance of these students. This paper reviews this literature within the Spanish context in order to assess its contributions, limitations and gaps. Despite the advanc-es, it is concluded that the absence of research that si-multaneously measures the proficiency in the language of instruction and the academic performance precludes rigor-ous empirical knowledge of such relationship and, hence, the contrast of the aforementioned hypothesis. The size of both the samples and the subsamples of the national origin groups, as well as a proper control for certain relevant vari-ables, are pointed out as other research challenges.

\section{KEYWORDS}

Children of immigrants; Educational achievement; Linguistic integration; Spanish language proficiency. 


\section{INTRODUCCIÓN}

La transición de España de país de emigración a país de inmigración provocó que, en un corto periodo de tiempo, el sistema educativo experimentara un importante proceso de diversificación cultural, especialmente en aquellas zonas de mayor densidad inmigratoria. Como resultado, el interés por las cuestiones que vinculan la inmigración y la educación pasó a un primer plano (Alegre 2008). Y entre estas, la relación existente entre la competencia lingüística del alumnado de origen extranjero y su integración en términos de rendimiento y logro académico se convirtió en uno de los principales focos de atención. Así ocurrió en la esfera política, en la escolar y en la académica.

En el ámbito político, las comunidades autónomas han otorgado a la enseñanza de la lengua de instrucción el papel más destacado dentro de las medidas dirigidas a la integración de esta población (Terrén 2008). Con diferentes denominaciones y modos de funcionamiento, casi todas han implementado aulas de apoyo lingüístico (Rahona y Morales 2013), las cuales han acaparado los mayores esfuerzos por parte de las administraciones autonómicas.

En el ámbito escolar, los niveles de competencia en la lengua vehicular han sido identificados también por algunos trabajos como el elemento que más preocupación genera a los docentes a propósito de las transformaciones que ha supuesto la inmigración (Navarro et al. 2012).

Y en el ámbito académico -en el que se centra este trabajo-, desde finales de los noventa han proliferado las publicaciones que prestan atención a la cuestión lingüística, convirtiéndose esta en uno de los principales objetos de estudio en lo que al binomio inmigración-educación se refiere (García Castaño, Rubio y Bouachra 2015). Muchas de ellas, y como ocurre también desde los otros dos ámbitos comentados, asumen de inicio que el conocimiento de la lengua escolar resulta esencial para el desarrollo educativo de este alumnado. En su tratamiento de la cuestión del rendimiento académico, esto se traduce en la defensa de la hipótesis de la desventaja lingüística (HDL de aquí en adelante) como elemento clave para explicar el inferior rendimiento de los hijos de inmigrantes (véase, por ejemplo: Navarro et al. 2012; Etxeberría 2005; Fullana, Vilá y Besalú 2003; Bullejos 2002; Siguan 1998; Díaz-Aguado, Baraja y Royo 1996).

Pero, después de tanta atención recibida, ¿en qué punto se encuentra la literatura académica en España en cuanto al estudio de la relación entre inmigración, lengua y rendimiento educativo? Más concretamente:

1) ¿Qué sabemos acerca del nivel de los hijos de inmigrantes en el español $u$ otras lenguas oficiales del Estado? ¿Cómo ha sido medido dicho nivel?;
2) ¿Cuál es el estado de la cuestión respecto al rendimiento académico de los hijos de inmigrantes? ¿Qué sabemos de sus diferenciales respecto a los nativos?; y, sobre todo,

3) ¿Qué hallazgos hay sobre cómo y en qué medida se ven afectados dichos diferenciales por el dominio de la lengua escolar?

Este trabajo pretende dar respuesta a tales preguntas mediante una revisión sistemática y crítica de las literaturas que las han abordado en el marco español. El objetivo principal es examinar y valorar su contribución al conocimiento empírico de estas cuestiones, señalándose tanto los avances realizados como sus limitaciones y lagunas. Conviene aclarar que, por razones de espacio y concreción, no serán objeto de este artículo los trabajos que analizan el bilingüismo, el cual añade otras aristas a las cuestiones previamente formuladas - ver Medvedeva y Portes (2016) para un estudio reciente sobre la relación entre bilingüismo y rendimiento en los hijos de inmigrantes en España-.

Si bien ya existen trabajos que han revisado parcialmente estas literaturas (García Castaño, Rubio y Bouachra 2015; Huguet y Janés 2013; Rahona y Morales 2013; Navarro et al. 2012; Vila 2011; Huguet y Navarro 2006), encontramos que estos adoptan un enfoque eminentemente descriptivo y en ellos no se hace explícito el método seguido. Sin negar su valor, el estado de la cuestión que aquí se presenta pretende aportar un carácter más crítico, así como sumar en exhaustividad y sistematicidad desde criterios de interdisciplinariedad.

El trabajo se estructura del siguiente modo. Primero, se sintetizan algunos de los más relevantes posicionamientos teóricos que han tratado el papel de la competencia lingüística en el rendimiento académico de los descendientes de inmigrantes. En segundo lugar, se contextualiza el caso español en términos de inmigración y lenguas de origen. En tercer lugar, se especifica el método seguido en el procedimiento de revisión bibliográfica. En cuarto lugar, se repasa y analiza críticamente la literatura que ha medido empíricamente el dominio de la lengua escolar por parte del alumnado de padres inmigrantes en este país. Seguidamente, se hace lo propio con los estudios que han tratado de avanzar en el conocimiento del rendimiento académico de este alumnado en España y, a continuación, con la literatura que ha tratado la relación entre esas dos variables: dominio de la lengua escolar y rendimiento.

\section{Consideraciones teóRICAS}

La lengua ha tenido un papel central en las explicaciones de las diferencias de rendimiento académico en general y de las existentes entre hijos de inmigrantes y de nativos en particular (MacSwan y Rolstad 2010). El argumento de base puede resultar 
obvio: si el dominio de la lengua escolar es esencial para el desarrollo educativo de todo niño, será entonces un elemento diferencial en el caso de chicos con padres que no son nativos de esa lengua. Pero, más allá de esto, podemos encontrar distintas consideraciones con respecto al factor lingüístico.

Las hallamos, por una parte, en las distintas perspectivas que, desde las ciencias sociales, han teorizado sobre los procesos de integración económica y social de los hijos de inmigrantes. Según la visión que tengan del resultado de esos procesos, tales perspectivas oscilan entre optimistas (en las que se encuadran la teoría de la asimilación lineal, la hipótesis de la ventaja de la segunda generación y la hipótesis del optimismo inmigrante), pesimistas (entre las que destaca la hipótesis de las generaciones de exclusión) y mixtas (en las que se sitúan la perspectiva de la edad de la migración y la teoría de la asimilación segmentada) (Portes y Rivas 2011; Kao y Tienda 2005).

Por otra parte, desde la lingüística y la psicología evolutiva, el estudio de la relación entre las competencias lingüísticas de los descendientes de inmigrantes y su rendimiento académico está fuertemente marcado por los trabajos de Jim Cummins, desarrollados inicialmente en Canadá. Nos detendremos más en ellos por su gran influencia sobre una de las literaturas aquí revisadas. Especial peso ha tenido la distinción entre BICS y CALP (Cummins 1981). Las BICS (Basic Interpersonal Communicative Skills) serían las habilidades lingüísticas conversacionales, necesarias para el uso de la lengua en situaciones informales. La CALP (Cognitive Academic Language Proficiency) sería la dimensión de las competencias lingüísticas relacionadas con las habilidades cognitivo-académicas, implicadas en situaciones formales como las que se dan en un contexto académico. Para Cummins (2002), aunque los alumnos hijos de inmigrantes adquieren rápidamente las BICS en la lengua dominante de la sociedad de llegada, les hacen falta como mínimo entre cinco y siete años para alcanzar el nivel de los hablantes nativos en la CALP. Estos resultados han encontrado soporte empírico a través de otros muchos estudios, tanto en el contexto internacional como en el español (Vila 2011). Como se verá más adelante, en este último la influencia académica de la obra de Cummins es patente.

Sin embargo, este tipo de resultados y la distinción BICS/CALP también han estado expuestos a importantes críticas (Wiley 1996; Martin-Jones y Romaine 1986; Rivera 1984; Edelsky et al. 1983). Dos de ellas son de especial relevancia para los propósitos de este trabajo.

La primera plantea que la popular distinción de Cummins confunde habilidad o competencia lingüística con conocimiento y rendimiento académico (MacSwan y Rolstad 2003; 2010). Cummins (1981) concibe las destrezas en lectura y escritura como aspectos del dominio de una lengua y, más concretamente, como componentes de la CALP. Sus críticos argumentan que estos, y el resto de elementos cognitivo-académicos incluidos en la CALP, son en realidad conocimientos y prácticas culturales desarrolladas fundamentalmente en el campo escolar. Desde esta óptica, la medición del dominio de una lengua en los términos de CALP resulta problemática, pues, del modo en que se conceptualiza, esta dimensión lingüística se solaparía con el conocimiento y el rendimiento académico. Y resulta más problemática aún si dicha medición es empleada para analizar la influencia de ese dominio lingüístico sobre el rendimiento académico. Teniendo esto en cuenta, el contraste de la validez de los instrumentos para medir la competencia lingüística pasaría necesariamente por demostrar ausencia de colinealidad con el rendimiento académico.

La segunda crítica conecta parcialmente con la anterior, al entender que el marco teórico de Cummins adolece de un entendimiento riguroso y profundo del amplio contexto social y cultural en el que el lenguaje se desarrolla y se practica (Martin-Jones y Romaine 1986; Genesee 1984; Troike 1984), y que no tiene en cuenta suficientemente los factores sociales que condicionan el rendimiento académico (Rivera 1984). En esta línea, se critica que ni en la conceptualización "BICS/CALP" ni en su medición se tengan en cuenta variables relevantes que afectan al dominio lingüístico y que estarían mediando en la relación entre el hecho de ser inmigrante (o de no ser nativo en una lengua) y dicho dominio (MacSwan y Rolstad 2010). Variables como los años de escolarización en el país de destino y en el de origen y, especialmente, las relativas al origen socioeconómico y sociocultural de los estudiantes. Tanto desde la sociolingüística como desde la sociología se han desarrollado explicaciones que enfatizan el papel del origen social en la adquisición de competencias o de registros lingüísticos. Un ejemplo es la distinción de Bernstein (1971) entre código elaborado y restringido. Pero, por encima de todo, la relevancia de estas variables está fundamentada empíricamente. Por un lado, el origen social se sitúa desde hace décadas entre los mejores predictores del rendimiento académico, tanto para la población nativa (Breen y Jonsson 2005) como para la inmigrante (OECD 2011; Heath, Rothon y Kilpi 2008; Levels, Dronkers y Kraaykamp 2008). Por otro, los hijos de inmigrantes suelen estar, por término medio, en desventaja con respecto al alumnado nativo en relación con estas variables. De ahí la importancia de que su efecto sea tenido en cuenta.

Pese a que Cummins ha respondido en más de una ocasión a estas y otras críticas matizando algunos de los aspectos de su teoría y de su distinción BICS/CALP (Cummins 2008; 1984), el uso de la misma en su concepción original se ha extendido considerablemente. Por tanto, el hecho de tener presente estas críticas es relevante no solo para el análisis de los trabajos de Cummins, sino también para el de aquellos que, de alguna manera, han tratado de replicar sus resultados en otros contextos. 


\section{CONTEXTO: LENGUA E INMIGRACIÓN EN ESPAÑA}

Desde la segunda mitad de la década de los noventa hasta 2008, España vivió un boom inmigratorio sin parangón entre los países de la OCDE (CebollaBoado y González-Ferrer 2008). Atraídos por el notable crecimiento de la demanda de trabajo, los nacidos fuera de España pasaron de ser menos de un millón y medio a sumar más de seis millones; del $2 \%$ al $16 \%$ del total de la población en edad de trabajar.

Hasta antes de iniciarse la última crisis económica internacional, la Encuesta Nacional de Inmigrantes de 2007 ofrecía la mejor fotografía sobre la composición de esa población. Los inmigrantes procedentes de Latinoamérica suponían algo más de un tercio $(37,2 \%)$. Les seguían los originarios de Europa (31,6\%), África $(13,1 \%)$ y otras regiones (18\%) (Reher y Requena 2009). En el plano lingüístico, el $45 \%$ indicaba el español como lengua de origen. Les seguían las lenguas indoeuropeas $(19,4 \%)$, las derivadas del latín distintas al español $(18,2 \%)$, las afroasiáticas $(11,6 \%)$, las orientales $(1,4 \%)$, africanas $(0,8 \%)$ y otras $(3,6 \%)$.

En el sistema educativo, el cambio fue igualmente intenso. El alumnado extranjero suponía en el curso $1997-98$ un $1 \%$ del total, mientras que diez años después representaba un 9,4\% (gráfico 1). El crecimiento continuó hasta el curso 2008-09, cuando alcanzó su máximo histórico (9,8\%). Entonces, la composición por grupos de nacionalidades no difería demasiado de la presentada para el conjunto de la población inmigrante en 2007. Los escolares procedentes de Latinoamérica representaban un $44 \%$ del alumnado extranjero, los europeos un $29 \%$, los africanos un $20 \%$ y los asiáticos un $5 \%$.
Pero la crisis económica iniciada a finales de 2007 precipitó el fin de la década prodigiosa de la inmigración en España (Arango 2009). A partir del curso 2009-10, el descenso de la población inmigrante comenzó a acusarse en el sistema educativo y el porcentaje de alumnado extranjero bajó levemente cada año hasta llegar al 8,4 \% de 2015-16. Desde entonces, los datos muestran una nueva y ligera tendencia al alza. No obstante, se ha de tener en cuenta que estas estadísticas emplean la variable "nacionalidad", por lo que subestiman el porcentaje de hijos de inmigrantes, al no incluir a aquellos que poseen la nacionalidad española. Asimismo, no se ha de olvidar que, aunque esta tendencia es generalizada para el conjunto del país, tanto el volumen de la población inmigrante como su composición por origen geográfico varían fuertemente entre comunidades autónomas y entre municipios.

Por grupos de nacionalidades, el mencionado descenso se concentró en los latinoamericanos, que en el curso 2017-18 pasaron a representar un $23 \%$ del total de este alumnado. En contraposición, han ganado peso los nacionales de países europeos, que suponen un $34 \%$, y especialmente los africanos (31 $\%)$ y asiáticos (11\%). Por tanto, si en relación con el objeto de estudio de este trabajo atendemos únicamente al origen geográfico del alumnado, puede concluirse que, por efectos composicionales, es en los años posteriores a 2010 cuando puede pensarse que la influencia de este factor lingüístico ha podido acentuarse. En años previos, el gran peso relativo que suponían los estudiantes procedentes de países hispanohablantes debía hacer que, estadísticamente, el desconocimiento del español fuese un factor menos relevante para explicar la desventaja académica general de los hijos de inmigrantes.

\section{Gráfico 1.}

Porcentaje de alumnado extranjero en el sistema educativo español (enseñanzas no universitarias)

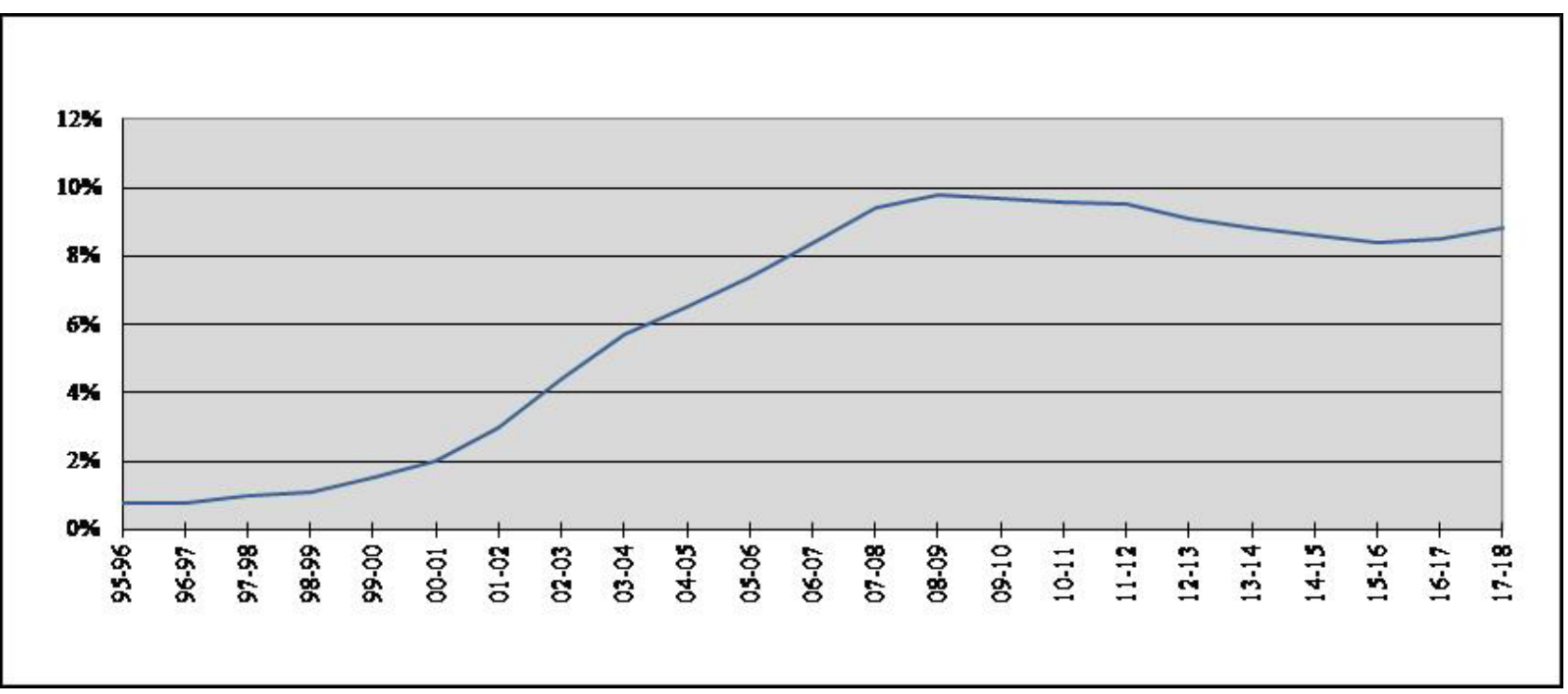

Fuente: Ministerio de Educación (2018). 


\section{Metodología}

Realizar un estado de la cuestión conlleva un proceso de construcción y de selección en el que han de hacerse explícitos los objetivos iniciales de la revisión, el criterio de selección aplicado y las conclusiones a las que se llega (Foster y Hammersley 1998). A la hora de seleccionar los trabajos a incluir en una revisión de la literatura se pueden aplicar dos estrategias diferenciadas (Gough, Thomas y Oliver 2012). Por un lado, la denominada estrategia agregativa, en la que el criterio de inclusión se establece a priori y que pretende ser lo más exhaustiva posible con objeto de reducir sesgos de selección. Aquí se incluyen las denominadas "revisiones sistemáticas" (Sánchez-Meca y Botella 2010). Por otro lado, están las revisiones basadas en estrategias de búsqueda y selección más configurativas, encaminadas a explorar y encontrar patrones o conclusiones relevantes en la literatura sobre un tema concreto, siendo lo importante detectar la relevancia de una contribución específica a la hora de testar una hipótesis o una teoría.

En este trabajo se aplican ambas estrategias. En primer lugar, puesto que el objetivo de partida era analizar el soporte empírico de la HDL en la literatura sobre el caso español, se realizó una revisión sistemática de los estudios que examinan la relación entre el dominio de la lengua escolar y el rendimiento académico de los hijos de inmigrantes en este país. Ello limitaba necesariamente la selección a trabajos empíricos que contuvieran mediciones de ambas variables. Se tuvieron en cuenta tanto mediciones objetivas como subjetivas. Por lo demás, se establecieron otros criterios de inclusión amplios: que fuesen trabajos científicos publicados en cualquier formato; escritos en inglés, español o cualquiera de las otras lenguas oficiales en España, y comprendidos entre 1995 y el momento de realización de la búsqueda, mayo de 2019 (ver cuadro 1).
La estrategia de búsqueda fue triple, comportando las siguientes fases:

$1^{\text {a }}$ Localización mediante los motores de búsqueda de las bases de datos WOS y Scopus. Los parámetros exactos de la búsqueda (rastreados en el título, abstract y palabras clave) pueden verse en la tabla 1.

$2^{a}$ Localización a través de trabajos de revisión bibliográfica o que contuviesen una amplia revisión bibliográfica. Concretamente, se consultaron los trabajos de García Castaño, Rubio y Bouachra (2015), Huguet y Janés (2013), Navarro et al. (2012), Vila (2011) y Huguet y Navarro (2006).

$3^{a}$ Búsqueda específica en otras revistas españolas no indexadas en WOS ni en Scopus en ese momento pero que, por su enfoque, podían haber publicado artículos en este campo. En concreto, se revisaron Lengua y Migración (no incorporada a Scopus hasta 2015), Migraciones (no incorporada a Scopus hasta 2012) y la Revista de la Asociación de Sociología de la Educación.

La búsqueda y selección de los trabajos aplicando los criterios de inclusión-exclusión fueron realizadas por dos personas de manera independiente, buscando asegurar una mayor precisión en el proceso.

En contraste con el ya citado elevado interés académico, político y escolar suscitado por esta cuestión en España, esta primera revisión arrojó una paradoja: la literatura empírica centrada en la relación entre el conocimiento de la lengua escolar y el rendimiento académico de los hijos de inmigrantes era muy escasa, tal y como se muestra en el tercer apartado de la sección de resultados. Esto nos llevó a revisar, en una segunda fase, otras dos literaturas para el caso español: la que analiza el nivel de los hijos de inmigrantes en la lengua vehicular y la que estudia el rendimiento académico de los hijos de inmigrantes. Estimábamos que solo así era posible

\section{Cuadro 1.}

Criterios de inclusión y exclusión para la revisión de la literatura que examina la relación entre el dominio de la lengua escolar y el rendimiento académico de los hijos de inmigrantes

\begin{tabular}{|l|l|l|}
\hline & \multicolumn{1}{|c|}{ CRITERIOS DE INCLUSIÓN } & \multicolumn{1}{c|}{ CRITERIOS DE EXCLUSIÓN } \\
\hline Población & $\begin{array}{l}\text { Estudiantes con al menos un padre/madre inmigrante } \\
\text { de cualquier etapa educativa en España } \\
\text { Estudiantes con ambos padres españoles tomados } \\
\text { como "grupo de control". }\end{array}$ & Resto de población. \\
\hline Diseño metodológico & $\begin{array}{l}\text { Estudios empíricos (con medidas objetivas o subjetivas } \\
\text { del dominio de una lengua oficial en España y del ren- } \\
\text { dimiento académico). }\end{array}$ & Estudios no empíricos (narrativos o especulativos). \\
\hline Idiomas & $\begin{array}{l}\text { Inglés, español y cualquiera de las otras lenguas oficia- } \\
\text { les en España. }\end{array}$ & Resto de idiomas. \\
\hline Rango temporal & 1995-mayo de 2019. & Antes de 1995. \\
\hline Tipo de publicación & Cualquier tipo. & - \\
\hline
\end{tabular}

Fuente: Elaboración propia. 
alcanzar una comprensión global del estado de la cuestión de nuestro objeto de estudio y entender mejor dicha paradoja. Para realizar estas otras dos revisiones se siguió una estrategia configurativa, con un protocolo de búsqueda y selección específico aunque flexible, en la línea de revisiones previas realizadas en esta área de estudio en otros países (Stevens 2007; Stevens et al. 2011). En este caso, partimos de revisiones bibliográficas elaboradas en trabajos previos. Para la primera de estas otras dos literaturas se volvieron a tener en cuenta los trabajos de García Castaño, Rubio y Bouachra (2015), Huguet y Janés (2013), Navarro et al. (2012), Vila (2011), y Huguet y Navarro (2006), mientras que para la segunda se partió de los de Álvarez-Sotomayor, Gutiérrez-Rubio y Martínez-Cousinou (2018) y Rahona y Morales (2013). Además de las publicaciones consideradas en estos trabajos de revisión, siguiendo la mencionada estrategia, se tuvieron en cuenta otras encontradas mediante búsquedas a través de WOS y Scopus, incorporando aquellas que contribuían a configurar un estado del arte en cada una de las dos cuestiones.

\section{Resultados}

\section{El nivel de los hijos de inmigrantes en la len- gua escolar}

En España encontramos un amplio número de trabajos que han abordado la comparación entre el dominio de la lengua escolar por parte de estudiantes hijos de inmigrantes y de nativos. Los clasificaremos aquí en tres grupos, en función del instrumento utilizado para la medición: los que emplean test lingüísticos, los que se basan en la autopercepción de los estudiantes y los que analizan la valoración del profesorado.

En el primer grupo encontramos, fundamentalmente, trabajos realizados por expertos en psicología evolutiva, lingüística y pedagogía. La mayor parte de ellos toman como referencia los estudios de Cummins y su explicada distinción entre BICS y CALP, y aplican pruebas diseñadas para medir las competencias lingüísticas de acuerdo con dicha distinción. Los resultados arrojan que los hijos de inmigrantes puntúan significativamente por debajo de los hijos de nativos en cuanto al conocimiento del español (Navarro, Huguet y Sansó 2014; González, Huguet y Chireac 2013; Navarro y Huguet 2010; Oller y Vila 2008; Huguet, Navarro y Janés 2007; Navarro y Huguet 2005; Mesa, Sánchez y Vázquez 1996), del catalán en aquellas investigaciones desarrolladas en Cataluña (Navarro, Huguet y Sansó 2014; González, Huguet y Chireac 2013; Navarro et al. 2012; Navarro y Huguet 2010; Oller y Vila 2008; Vila 2008) y del euskera en el País Vasco (véase Etxeberría et al. 2019). Además, cuando en estos trabajos se analizan estas puntuaciones en función del tiempo de residencia, algunos encuentran que este alumnado requiere un periodo considerable (más de seis años) para alcanzar el nivel del alumnado nativo en las competencias lingüísticas tipo CALP (Navarro et al. 2012; Navarro y Huguet 2005), resultados que se interpretan como un refuerzo a la tesis de Cummins. Otros hallan que esta equiparación no llega ni siquiera después de seis años de residencia (Oller y Vila 2008; Huguet, Navarro y Janés 2007).

Algunos de estos trabajos incluyen a hispanohablantes en sus muestras y los resultados van en la línea de los anteriores: pese a compartir el español como lengua materna, este alumnado también puntúa significativamente por debajo del de padres nacidos en España en las pruebas lingüísticas, aunque en algunos casos la desventaja es mucho menor que la que presentan los inmigrantes no hispanohablantes (Navarro, Huguet y Sansó 2014; Navarro et al. 2012; Huguet, Navarro y Janés 2007; Navarro y Huguet 2005).

En cuanto al conocimiento lingüístico de los Ilamados "inmigrantes de segunda generación", los resultados de algunos de estos estudios muestran que su nivel mejora sustantivamente con respecto a la primera generación, si bien aún se sitúan por debajo de los hijos de nativos (Arjona, Checa y Checa 2014; Navarro et al. 2012). La segunda generación es conceptualizada en estos trabajos como los hijos de inmigrantes nacidos en España.

Los trabajos que pueden incluirse en el segundo grupo —basados en la autopercepción lingüística del alumnado - tienen mucha menos presencia en la literatura centrada en España. De hecho, en esta revisión solo se han hallado dos, los cuales, además, presentan resultados enfrentados. De una parte, en la investigación de Álvarez de Sotomayor (2011), y en contraste con los resultados del primer grupo de trabajos, tanto la segunda generación (operacionalizada como los nacidos en España o llegados antes de los seis años de edad), como los descendientes de oriundos de países hispanohablantes muestran un nivel parejo de español al de los hijos de nativos. Esto supone cerca de un $80 \%$ de la población analizada (alumnado de $3 .^{\circ} \mathrm{y}$ $4 .^{\circ}$ de ESO de los institutos de Marbella). En este sentido, la desventaja lingüística se concentra en el alumnado de primera generación, que, no obstante, alcanza el nivel de sus pares nativos cuando lleva más cinco años residiendo en España. De otra parte, Siguan (1998) destaca que, en su estudio de caso (ocho centros de EGB de Madrid y Barcelona), la mayoría de estudiantes hijos de inmigrantes estarían en situación de desventaja lingüística en relación con la lengua vehicular del sistema educativo, si bien no se cuantifica ni la desventaja ni la proporción que supone esa mayoría. 
Finalmente, en los trabajos que analizan las valoraciones y percepciones del profesorado sobre el dominio de la lengua escolar tampoco hay resultados completamente uniformes. Algunos encuentran que el profesorado percibe un aprendizaje relativamente rápido en la mayor parte de este alumnado; valoración registrada tanto mediante técnicas cuantitativas (Díaz-Aguado, Baraja y Royo 1996), como cualitativas (Álvarez de Sotomayor 2011; Colectivo IOÉ 1996). Incluso cuando el profesorado discierne el ritmo de aprendizaje en la adquisición de habilidades básicas de comprensión y expresión, por un lado, y de habilidades lingüísticas de carácter más académico, por otro, establece que para las primeras la media se sitúa en menos de cuatro meses y para las segundas alrededor de un año y medio (Díaz-Aguado, Baraja y Royo 1996), tiempos muy por debajo de los detectados en el primer grupo de trabajos. Por el contrario, Siguan (1998) relata que la mayoría de los niños y niñas inmigrados de su caso de estudio presenta un déficit, mayor o menor, en el idioma de instrucción.

En cuanto a las limitaciones de estos estudios, si lo que se quiere analizar es el nivel de dominio de una lengua, la forma idónea de hacerlo es mediante pruebas objetivas. En consecuencia, y a pesar de la existencia de literatura que avala la utilización de la autopercepción del nivel lingüístico como proxy del nivel objetivo que se posee (Stolzenberg y Tienda 1997; Hakuta 1986), los trabajos incluidos en los dos últimos grupos presentan como primera y principal limitación el cuestionamiento sobre su fiabilidad. En el apartado en que se revisa la literatura que ha abordado la relación entre el dominio de la lengua escolar y el rendimiento académico se exponen otras observaciones críticas.

Por su parte, los incluidos en el primer grupo tienen, a nuestro entender, limitaciones ya presentes en algunas de las comentadas críticas a la obra de Cummins. Es decir, en primer lugar, puede surgir la duda de hasta qué punto miden únicamente la habilidad lingüística o si miden también conocimiento académico. El hecho de que se centren fundamentalmente en el dominio formal de la lengua -baremando, además de la comprensión oral y escrita, los conocimientos en morfosintaxis, ortografía y entonación lectora entre otros, es decir, conocimientos adquiridos en el entorno escolar (ver, por ejemplo, Huguet 2008) - refuerza esta duda. Para disiparla se requeriría, en todo caso, que los instrumentos de medición empleados hubiesen demostrado ausencia de colinealidad con el rendimiento académico, algo que no hemos detectado. En segundo lugar, estos estudios presentan importantes carencias a la hora de tener en cuenta variables relevantes que pueden mediar en la relación entre el hecho de ser hijo de inmigrante y el dominio que se posea en la lengua escolar. Así, por ejemplo, ni el origen social del alumnado ni los años de escolarización previos son adecuadamente controlados, cuando no obviados. Una excepción es el mencionado trabajo de Arjona, Checa y Checa (2014), que introduce en modelos de regresión variables como la generación migratoria, el género, la religión, los años de escolarización en España y los ingresos. El mayor problema de este trabajo es que los análisis se realizan únicamente sobre hijos de inmigrantes, no incluyéndose en la muestra al alumnado nativo.

Además, la gran mayoría de estas publicaciones parten de investigaciones que trabajan con muestras de población pequeñas $y$, consecuentemente, con submuestras de orígenes nacionales (o de inmigrantes agrupados según familias lingüísticas de sus lenguas de origen) muy reducidas. Ello conlleva problemas de representatividad estadística en ambos niveles.

\section{El rendimiento académico de los hijos de in- migrantes}

En el panorama internacional, los datos muestran que los hijos de inmigrantes tienen, de media, un inferior rendimiento académico que los hijos de nativos (Waters et al. 2013; OECD 2011, 2016; Heath, Rothon y Kilpi 2008; Stanat y Christensen 2006). Igual ocurre en un país de corta trayectoria inmigratoria como España, donde esta desventaja general es ya una regularidad bien documentada a partir indicadores muy diversos: tasas de abandono o de escolaridad en secundaria y en la etapa postobligatoria (Serra y Palaudàrias 2010; Aparicio y Tornos 2006; Colectivo IOÉ 2003; Fullana, Vilá y Besalú 2003), tasa de idoneidad (Defensor del Pueblo 2003), calificaciones del alumnado (Álvarez de Sotomayor 2011), dificultades en el aprendizaje al finalizar Primaria (Fullana, Vilá y Besalú 2003), pruebas de diagnóstico en Primaria (Cebolla-Boado y Garrido 2008) y resultados en las competencias evaluadas por PISA (por ejemplo: OCDE 2016; Schnell y Azzolini 2015; Zinovyeva, Felgueroso y Vázquez 2013; Salinas y Santín 2012; Cebolla-Boado 2008; Calero, Choi y Waisgrais 2009). Y aunque los datos de esta última fuente apuntan a la existencia de grandes diferencias entre comunidades autónomas, solo el caso canario parece desviarse de la norma de la desventaja inmigrante (Álvarez-Sotomayor, Gutiérrez-Rubio y Martínez-Cousinou 2018).

En lo referente a la búsqueda de explicaciones, en España se evidencia la importancia de la desigualdad socioeconómica como factor explicativo de tal desventaja (Álvarez-Sotomayor y Martínez-Cousinou 2016; Schnell y Azzolini 2015; Zinovyeva, Felgueroso y Vázquez 2013; Calero, Choi y Waisgrais 2009), resultado en línea con lo hallado en la literatura internacional (Levels, Dronkers y Kraaykamp 2008). Aun así, y como ocurre también en otros países europeos (Heath, Rothon y Kilpi 2008), el origen social no explica más del $50 \%$ o el $60 \%$ de la varianza, lo que deja lugar para otro tipo de explicaciones. 
Más allá del origen social, se han explorado los efectos de otras variables individuales y escolares. Entre las individuales, se ha hallado sistemáticamente que el efecto de ser de segunda generación es positivo y fuerte. Las primeras generaciones puntúan claramente por debajo de los nativos (Calero, Choi y Waisgrais 2010; González-Betancort y López-Puig 2008), siendo la desventaja de las segundas generaciones sustancialmente menor (OECD 2016; Zinovyeva, Felgueroso y Vázquez 2013; Salinas y Santín 2012) o incluso no significativa (Calero, Choi y Waisgrais 2009; González-Betancort y López-Puig 2008). Y entre la primera generación, la edad de llegada y de escolarización en España también se halla como un factor de peso (Colectivo IOÉ 2003).

Una segunda variable empleada como indicador del estatus migratorio es aquella que distingue si el padre y la madre son inmigrantes o si solo lo es uno de los dos, habiendo nacido el otro en España (parejas mixtas). En los países occidentales se halla un fuerte efecto positivo del hecho de provenir de parejas mixtas (Rindermann y Thompson 2016). En España, los análisis de PISA confirman esta tendencia; tanto que la desventaja en relación con el alumnado nativo desaparece en este grupo (Álvarez-Sotomayor, Gutiérrez-Rubio y Martínez-Cousinou 2018; Cebolla-Boado y González 2008).

Como se detalla más adelante, debido a la escasez de datos adecuados, menos concluyente es el conocimiento respecto a una variable esencial: el origen nacional o étnico. Sabemos que el rendimiento varía sustantivamente en función de esta variable (Portes, Aparicio y Haller 2016; Álvarez de Sotomayor 2011; Cebolla-Boado y Garrido 2008; Aparicio y Tornos 2006; Colectivo IOÉ 2003), pero aún son necesarios más y mejores datos para alcanzar un conocimiento más sólido.

En relación con los factores escolares, los resultados apuntan a que su impacto sobre el diferencial entre hijos de inmigrantes y de nativos es limitado (Álvarez de Sotomayor 2011; OECD 2010; Calero, Choi y Waisgrais 2009; Carabaña 2008). Los resultados sobre la influencia de la concentración de alumnado inmigrante -la variable escolar que ha acaparado mayor atención académica y mediática en este asunto- no son concluyentes, con trabajos que encuentran un efecto negativo y otros que no detectan efecto alguno (Carabaña 2016). Por su parte, diversos trabajos de corte cualitativo (por ejemplo: Ballestín 2015; Rubio Gómez 2013; García Castaño y Olmos 2012; Pàmies 2006) permiten pensar que determinadas representaciones sociales estereotipadas, halladas algunas entre alumnado y otras entre profesorado, y construidas a partir de categorizaciones raciales, culturalistas, nacionales o basadas en el tiempo de residencia (Álvarez-Sotomayor 2015), pueden tener algún peso como desencadenantes de procesos discriminatorios.
Pese a los avances señalados, la investigación empírica sobre esta cuestión en España se encuentra en una situación de notable atraso en comparación con países de mayor tradición inmigratoria. La ausencia de datos de calidad para el estudio de esta cuestión (Cebolla-Boado 2008) impone limitaciones sustanciales. Destacamos algunas de ellas:

1. Problemas de representatividad estadística. Gran parte de los trabajos que nutren esta literatura algunos de ellos de importante valor descriptivo y explicativo- se circunscriben a contextos muy reducidos: estudios de caso de uno o unos pocos centros o, a lo sumo, de un municipio. La gran excepción se halla en las explotaciones de los datos de PISA, representativos de todo el país y con submuestras representativas también de la mayoría de CC. AA. desde 2009. Sin embargo, PISA posee la siguiente limitación.

2. Problemas con los datos de orígenes nacionales. En España, PISA cuenta con la importantísima limitación de no distinguir el origen nacional del alumno; solo permite conocer si este es de origen nativo o no. La limitación es de suma importancia porque está ampliamente demostrado que el rendimiento varía fuertemente según el origen nacional, que se muestra, de hecho, más determinante que los factores vinculados al destino (Carabaña 2011).

Por su parte, los estudios de caso ya señalados arrastran los problemas derivados del habitual reducido tamaño de sus muestras al tamaño de las submuestras de los grupos de origen, lo cual limita los análisis desagregados por esta variable. Otras veces, estos estudios se centran únicamente en uno o en un número reducido de grupos de origen (por ejemplo: Aparicio y Tornos 2006; Colectivo IOÉ 2003; Fullana, Vilá y Besalú 2003), lo que, si bien puede tener un notable valor añadido por la profundidad de análisis que permite, imposibilita la comparación con el resto.

El resultado de todo esto es la ausencia en España de un conocimiento fiable sobre la estratificación del rendimiento académico en función del origen nacional. Los avances logrados a este respecto resultan todavía insuficientes.

3. Ausencia de otras variables relevantes. Ya se ha anotado que, más allá del origen social, la investigación sobre los condicionantes de este diferencial académico en España es aún poco concluyente. PISA, la fuente más empleada e importante para conocer esta cuestión, contiene una gran cantidad de variables de interés, pero no está específicamente diseñado para el estudio de la brecha académica entre hijos de inmigrantes y de nativos. Ello explica que no contemple otras que son señaladas por la literatura especializada como relevantes; principalmente, variables ligadas a explicacio- 
nes étnicas (Cebolla-Boado 2008). Por ejemplo, variables que midan la discriminación, diferencias culturales y el conocimiento de la lengua escolar, carencia esta última sobre la que se profundiza a continuación. Este vacío ha sido parcialmente cubierto por estudios de caso, los cuales, no obstante, presentan los problemas de validez externa ya señalados.

\section{La relación entre el dominio de la lengua es- colar y el rendimiento académico}

Como se detalla a continuación, de la revisión sistemática de la literatura especializada puede concluirse que, en España, la HDL ha sido contrastada escasa y limitadamente hasta el momento. En la tabla 1 se recoge el número de resultados obtenidos en cada una de las búsquedas realizadas en los motores de WOS y Scopus, así como los totales.

No obstante, tras la aplicación de los criterios de inclusión detallados en la metodología, el número de trabajos seleccionados se redujo a seis (ver el cuadro 2). Es decir, tras nuestra revisión encontramos tan solo seis trabajos que permiten a priori el contraste de la HDL en España. De ellos, dos fueron hallados a través de las búsquedas realizadas en WOS y Scopus (fase 1 de la búsqueda bibliográfica) y cuatro a través de las revisiones realizadas en otros trabajos (fase 2).

La razón principal del número tan escaso es que son todavía pocos los estudios que incluyan simultáneamente indicadores del conocimiento de los estudiantes en la lengua de instrucción y de su rendimiento académico. Ya hemos adelantado que constituye un problema el hecho de que PISA, la fuente más empleada para analizar el rendimiento de los estudiantes de origen inmigrante en España, no permita dicho contraste, pues solo contiene como variable lingüística la lengua hablada en el hogar. Aun así, cabe señalar que, a diferencia de lo hallado en otros contextos (Stanat y Christensen 2006), en España esta variable parece no afectar significativamente a los resultados de los hijos de inmigrantes (Calero, Choi y Waisgrais 2009; González-Betancort y López-Puig 2008).

Entre los trabajos seleccionados (ver el cuadro 2) destaca la ambiciosa investigación longitudinal de Portes, Aparicio y Haller (2016), con muestras representativas de las áreas metropolitanas de Madrid y Barcelona. En ella se halla que, entre los hijos de inmigrantes, el nivel (auto-percibido) de español mejora significativamente tanto sus calificaciones académicas como la probabilidad de permanecer escolarizado a los 18 años, incluso después de tener en cuenta diversas variables relevantes (sexo, estatus socioeconómico, si se nació en España, tiempo de residencia, años de escolarización, horas de estudio del alumno o alumna y estructura parental del hogar).
Tabla 1.

Número de documentos encontrados en WOS y Scopus según parámetros de búsqueda

\begin{tabular}{lrr}
\hline \multicolumn{1}{c}{ Ecuación de búsqueda } & WOS & SCOPUS \\
\hline Spanish + achievement + immigr ${ }^{*}+$ Spain & 30 & 18 \\
Language + achievement + immigr ${ }^{*}+$ Spain & 17 & 12 \\
Linguistic + achievement + immigr ${ }^{*}$ Spain & 5 & 4 \\
Spanish + attainment + immigr* + Spain & 26 & 25 \\
Language + attainment + immigr* + Spain & 9 & 5 \\
Linguistic + attainment + immigr ${ }^{*}+$ Spain & 1 & 3 \\
Spanish + performance + immigr + Spain & 55 & 45 \\
Language + performance + immigr ${ }^{*}$ Spain & 17 & 13 \\
Linguistic + performance + immigr + Spain & 10 & 9 \\
Spanish + achievement + foreign + Spain & 14 & 16 \\
Language + achievement + foreign + Spain & 17 & 12 \\
Linguistic + achievement + foreign + Spain & 3 & 0 \\
Spanish + attainment + foreign + Spain & 8 & 4 \\
Language + attainment + foreign + Spain & 4 & 0 \\
Linguistic + attainment + foreign + Spain & 2 & 0 \\
Spanish + performance + foreign + Spain & 75 & 61 \\
Language + performance + foreign + Spain & 29 & 27 \\
Linguistic + performance + foreign + Spain & 6 & 5 \\
Total & 242 & 193 \\
\hline
\end{tabular}

Fuente: Elaboración propia.

Nota: en el total solo se contabiliza una vez cada una de las publicaciones halladas.

Por el contrario, la variable lingüística no tiene un efecto significativo sobre la probabilidad de estar matriculado en bachillerato, en un ciclo superior o en la universidad (Portes, Aparicio y Haller 2016: 157-165). Pese al indudable interés de los resultados, la principal limitación en cuanto al objeto de estudio que aquí se plantea es que los análisis se realizan únicamente sobre muestras de hijos de inmigrantes, tomándose como grupo de referencia subgrupos de estos y no una muestra del alumnado nativo. Por lo tanto, lo que se analizan son los diferenciales entre distintos grupos de descendientes de inmigrantes y no entre estos y los hijos de nativos, que es la cuestión abordada en este artículo.

Por su parte, con un estudio de caso centrado en Marbella, Álvarez de Sotomayor (2011) encuentra que, una vez controlado el efecto del origen social, el factor lingüístico no ayuda a explicar la desventaja académica agregada del conjunto de los hijos de inmigrantes y tan solo explica una pequeña porción de los diferenciales de dos de los cinco grupos de origen distinguidos y, en ningún caso, en la asignatura de matemáticas. Aunque el dominio del español se muestra como una variable relevante para el rendimiento del alumnado, se interpreta que diversos efectos de composición (los derivados del alto porcentaje originario de países hispanohablantes y de la importante proporción que supone la segunda 
generación y quienes llevan más de cinco años residiendo en España) explicarían el hecho de que esta afecte poco o nada a las desigualdades académicas entre inmigrantes y nativos. No obstante, pese al interés de los resultados, la investigación presenta dos limitaciones relevantes. La primera, relativa a la utilización de la autopercepción del alumnado como indicador del conocimiento del español, ya comentada previamente y presente también en el trabajo de Portes, Aparicio y Haller (2016). Y la segunda, que el estudio se circunscribe a los institutos de secundaria del municipio de Marbella, lo que restringe su validez externa a ese ámbito.

Centrado en la etapa de Infantil encontramos el estudio de Calero et al. (2013), que analiza una muestra de solo 176 escolares de entre cuatro y cinco años en Granada. Incluye tres submuestras de niños con padres nativos, nacidos en países hispanohablantes y nacidos en países no hispanohablantes, respectivamente. Los hijos de inmigrantes son todos nacidos en España (segunda generación). El nivel lingüístico de los niños es evaluado por los propios investigadores tras su observación, mientras que el rendimiento se mide mediante los reportes de sus maestras y maestros en seis áreas: matemáticas, expresión oral y escrita, identidad personal y autonomía, entorno físico y social, desarrollo psicomotor y expresión artística. Los resultados no muestran diferencias significativas de rendimiento entre los tres grupos, por lo que la HDL no tiene cabida en este caso.

Como veremos, el resto de trabajos que pueden incluirse entre los intentos por avanzar en el conocimiento empírico de la relación entre conocimiento de la lengua escolar y rendimiento añaden al problema de la validez externa (muestras pequeñas realizadas sobre un número reducido de centros) diversas debilidades metodológicas y de otro tipo que hacen que la validez y fiabilidad de sus resultados sea cuestionable.

En la década de los noventa, Siguan (1998) analizó ocho centros de la entonces EGB (cuatro de Madrid y cuatro de Barcelona) y Díaz-Aguado, Baraja y Royo (1996) siete en Madrid. En ambos casos, se apuntaba la importancia del déficit lingüístico de los niños inmigrados como factor explicativo de sus peores resultados escolares. Como indicadores de rendimiento, el primero utilizó la tasa de idoneidad, las calificaciones en matemáticas y en lengua y la obtención o no del graduado escolar; y el segundo la valoración del profesor, si bien no se aporta información sobre cómo fue medida. En cuanto al conocimiento de la lengua de instrucción, en Siguan (1998) se midió a través de la percepción del propio alumnado y de la valoración del profesorado, mientras que Díaz-Aguado, Baraja y Royo (1996) utilizaron cuestionarios para conocer la percepción del profesorado al respecto.
Una primera limitación de estos dos estudios es precisamente el uso de las percepciones del profesorado como indicador y los posibles sesgos asociadas a estas. Recordemos, además, que también encontramos trabajos de corte cualitativo que presentan resultados opuestos, destacando que el profesorado relativiza el peso del factor lingüístico en la medida en que percibe que, a determinadas edades, la mayoría de estos chicos aprenden el idioma rápidamente (Álvarez de Sotomayor 2011; Colectivo IOÉ 1996). Un segundo problema de los estudios de Siguan (1998) y Díaz-Aguado, Baraja y Royo (1996) son las implicaciones derivadas del reducido tamaño de sus muestras, así como el hecho de haberse realizado en un momento anterior al cambio demográfico provocado por el boom inmigratorio descrito anteriormente. También lo es el hecho de que no se controlen los efectos de otras variables relevantes. Asimismo, en el trabajo de Siguan los resultados son solamente relatados, sin ofrecer explotación estadística alguna de los datos.

Por último, el trabajo de Blanchard (2009) se centra en un único instituto de Madrid, con una muestra también muy reducida. En este caso, se emplean los resultados en pruebas lingüísticas estandarizadas como indicador del domino del español, mientras que el rendimiento académico se mide a través de pruebas estandarizadas en matemáticas y lengua. En el trabajo se concluye que "la lengua es un factor fundamental en el aprendizaje" (Blanchard 2009: 145), apuntando a esta como variable explicativa del inferior rendimiento del "alumnado extranjero", una vez comprobado también su menor puntuación en la prueba lingüística. Sin embargo, para ello se basa únicamente en resultados de correlaciones bivariadas.

Este pobre estado de la cuestión contrasta con el amplio nivel de aceptación con el que parece contar la HDL, pues, como se ha comentado en la introducción, la explicación lingüística ha ocupado un lugar prevalente en el ámbito académico y en el político.

\section{Conclusiones}

En el análisis del binomio inmigración-educación en España, la cuestión del dominio de la lengua escolar ha sido un importante objeto de preocupación. Así ha ocurrido en las esferas escolar, política y académica. El motivo fundamental es la asunción de que el menor nivel de los hijos de inmigrantes en la lengua de instrucción es un factor clave para explicar su peor rendimiento académico general —hipótesis de la desventaja lingüística (HDL) - Con el fin de conocer el sustento empírico de esta hipótesis en el caso español, este artículo tenía como objetivo inicial realizar un estado de la cuestión de la literatura que había analizado empíricamente la relación entre el dominio de la lengua escolar y el rendimiento académico de este alumnado. La revisión sistemática de 


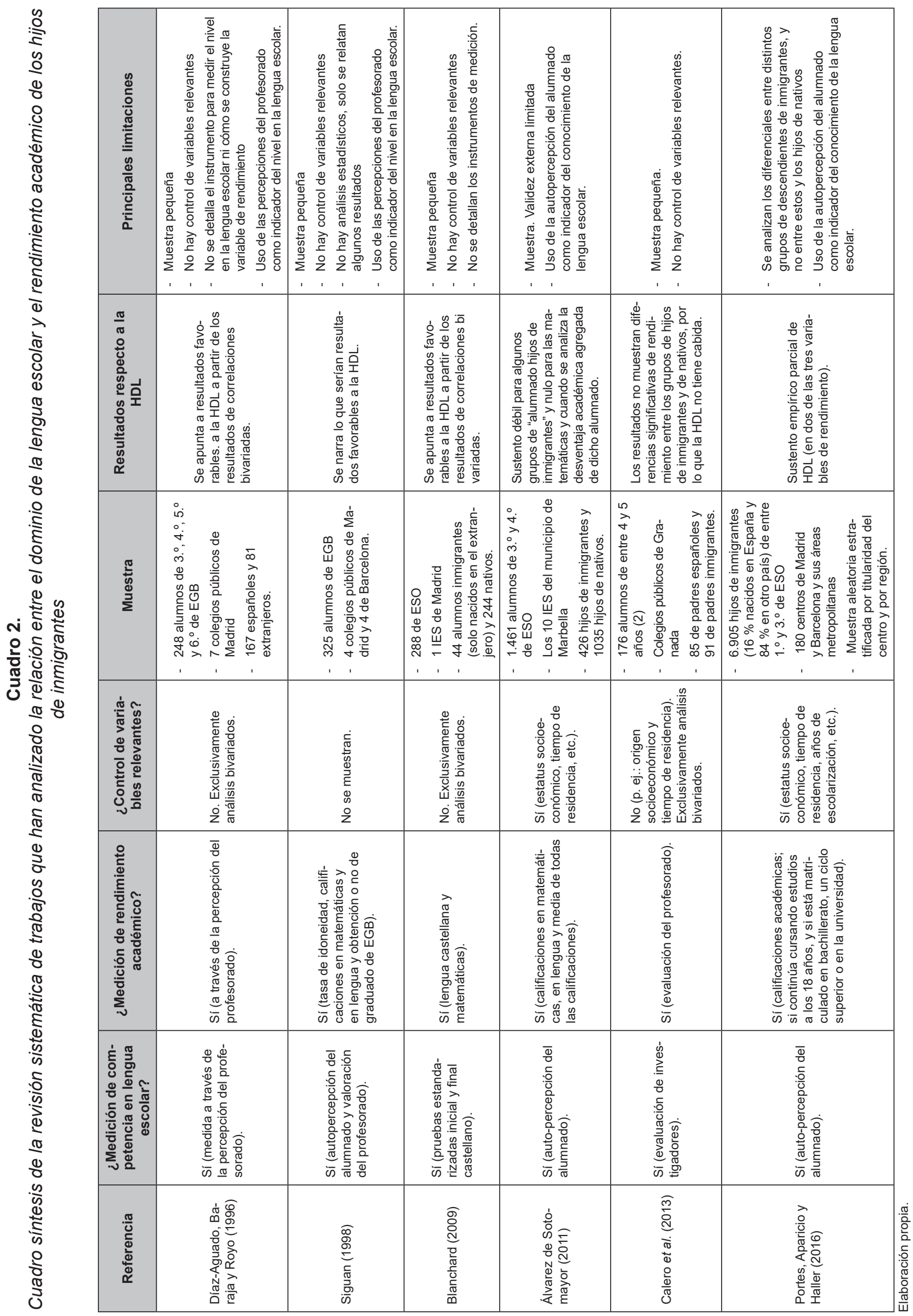


dicha literatura dio como resultado un número muy bajo de trabajos, lo cual, si bien era ya un dato significativo en sí mismo, entraba en contradicción con el apoyo mostrado a la HDL. Por ello, se consideró oportuno elaborar también por separado los estados de la cuestión de los dos elementos implicados en esa relación: el nivel de los hijos de inmigrantes en la lengua vehicular dentro del sistema educativo, por un lado, y el rendimiento académico de los hijos de inmigrantes, por otro, lo que suponía llevar a cabo las revisiones de otras dos literaturas. Entendíamos que, de esta forma, posibilitábamos una comprensión más amplia de nuestro objeto de estudio y de ese apoyo a la HDL.

Los resultados de estas tres revisiones nos llevan a concluir que, pese a los avances realizados, los tres ámbitos de la literatura abordados presentan importantes limitaciones que deberían ser tenidas en cuenta en las tres esferas previamente señaladas.

En cuanto al nivel de los hijos de inmigrantes en la lengua escolar, los trabajos que lo miden mediante pruebas estandarizadas concluyen que, si bien este alumnado obtiene rápidamente un nivel alto en las habilidades conversacionales, requiere de mucho más tiempo para alcanzar a sus pares nativos en las competencias lingüísticas relacionadas con las habilidades cognitivo-académicas. Los resultados apuntan que esto es así incluso para el alumnado con origen en países hispanohablantes. Sin embargo, limitaciones como el hecho de no controlar el efecto de otras variables relevantes, la restringida validez externa de estos estudios y no afrontar aparentemente las importantes críticas apuntadas sobre la distinción de Cummins entre BICS y CALP, advierten de la necesidad de llevar a cabo más investigaciones en este terreno.

En lo concerniente a los trabajos sobre el rendimiento académico diferencial de los hijos de inmigrantes en España, los avances han sido sustanciales. Ampliamente demostrada está la desventaja general de este alumnado, así como el efecto sobre la misma del origen socioeconómico, del estatus migratorio del alumnado y de determinadas variables escolares. Aun así, la escasez de datos cuantitativos de calidad para el estudio específico de esta cuestión (Cebolla-Boado 2008) se evidencia, entre otras, en las carencias existentes en los análisis en función del origen nacional y en las dificultades para contrastar las denominadas "explicaciones étnicas".

Por último, especialmente pobre es el estado de la cuestión relativo al conocimiento empírico de la relación entre el dominio de la lengua escolar y el rendimiento académico, aquel en el que debe sustentarse el contraste de la HDL. Fundamentalmente, debido a la escasez de investigaciones que midan conjuntamente ambas variables. Además, las existentes ofrecen resultados que están lejos de poder considerarse concluyentes. Primero, porque, como se ha visto, no son unánimes. Segundo, por sus limitaciones (medición del nivel en la legua escolar mediante la autopercepción del alumnado, problemas de validez externa por lo reducido de las muestras y otro tipo de debilidades metodológicas que ponen en cuestión la validez y fiabilidad de los resultados de estos trabajos). $Y$ tercero, por el bajo número que suponen todavía.

Como ya se ha destacado, este pobre estado de la cuestión contrasta con el alto grado de aceptación con el que ha contado la HDL en los ámbitos académico y político. Ante tal paradoja cabe preguntarse si existe algún otro tipo de aparente sustento empírico que, desde la academia, haya podido servir para valorar la relación entre el dominio de la lengua escolar y el rendimiento académico diferencial de los hijos de inmigrantes en España en el sentido apuntado por la HDL.

Una respuesta la hallamos en los resultados generales de las dos primeras literaturas revisadas en nuestro trabajo. Partiendo de ellos - por un lado, los déficits competenciales en la lengua de instrucción por parte de los hijos de inmigrantes y, por otro, su desventaja académica-, algunos trabajos (por ejemplo, Navarro et al. 2012; Huguet 2008; Huguet y Navarro 2006) sugieren, cuando no dan por hecha, la relación causal entre ambos tipos de variables. Obvian la exigencia de medir simultáneamente la competencia en el español y el rendimiento académico, así como las limitaciones de ambas literaturas. El siguiente extracto de un artículo de Navarro et al. (2012: 463), en el que se acaban citando estudios que solo miden las competencias lingüísticas, no el rendimiento académico, es un buen ejemplo de ello:

Evidentemente, estas dificultades en el uso de lenguaje [por parte de los hijos de inmigrantes] condicionan los resultados inferiores en el conjunto de aprendizajes y contenidos escolares y se traducen en un fracaso escolar muy superior al de sus pares autóctonos (Huguet y Navarro 2006; Huguet 2008;

Oller y Vila 2008; Vila 2008; Navarro y Huguet 2010).

Otro tipo de trabajos empíricos que puede haber alimentado la aceptación de la HDL son aquellos que, aunque no contienen mediciones del nivel lingüístico ni del rendimiento académico $-\mathrm{y}$, por ello, no han sido incluidos en esta revisión-, sí que registran las percepciones de agentes educativos sobre los condicionantes del rendimiento académico de los hijos de inmigrantes. Son los casos de las investigaciones de Franzé (2008), en la que el dominio del idioma vehicular es mencionado por docentes de ESO como uno de los factores más influyentes en el rendimiento de este alumnado, o Cano y Fernández (2015), en la que una muestra de 13 hijos de inmigrantes que han alcanzado la universidad consideraba la competencia comunicativa como una de las claves de su éxito académico. 
Más allá de esto, la HDL ha podido verse reforzada por trabajos de carácter meramente especulativo. Estos - tampoco incluidos en esta revisión- suelen apoyarse en resultados procedentes de otros países y contextos, o en lo que parece "sentido común". Nuestro trabajo evidencia que, por encima de todo ello, para contrastar esta hipótesis y conocer rigurosamente la relación entre el dominio de la lengua escolar y el diferencial académico entre hijos de inmigrantes y de nativos, se requieren más estudios

\section{REFERENCIAS BiBLIOGRÁfICAS}

Alegre, M. A. 2008. "Educación e inmigración. ¿Un binomio problemático?". Revista de Educación 345: 61-82.

Álvarez de Sotomayor, A. 2011. "El rendimiento académico de los alumnos inmigrantes en España: un estudio de caso". Tesis doctoral, Departamento de Sociología, Universidad de Granada.

Álvarez-Sotomayor, A. 2015. "El papel del origen nacional y del tiempo de residencia en los etiquetados profesorales de hijos de inmigrantes en secundaria". Revista de la Asociación de Sociología de la Educación 8(3): 380-395.

Álvarez-Sotomayor, A. y G. Martínez-Cousinou. 2016. “¿Capital económico o cultural? El efecto del origen social sobre las desventajas académicas de los hijos de inmigrantes en España". Papers: Revista de Sociología 101(4): 527-554. https://doi: 10.5565/rev/papers.2200

Álvarez-Sotomayor, A., D. Gutiérrez-Rubio y G. Martínez-Cousinou. 2018. "¿Desventaja académica de los hijos de inmigrantes? La excepción canaria en el contexto español". Revista Española de Sociología 27(1): 83-106. https://doi.org/10.22325/fes/res.2018.5

Aparicio, R. y A. Tornos. 2006. Hijos de inmigrantes que se hacen adultos: marroquíes, dominicanos, peruanos. Madrid: Ministerio de Trabajo.

Arango, J. 2009. "Después del gran boom: la inmigración en la bisagra del cambio." Pp. 52-73 en La inmigración en tiempos de crisis. Anuario de la inmigración en España, editado por E. Aja, J. Arango y J. Oliver. Barcelona: Fundació CIDOB.

Arjona, Á., J. C. Checa y D. Checa. 2014. "Extranjeros en Almería: lengua meta y código escrito". Álabe 10. http:// revistaalabe.com/index/alabe/article/view/206/179 [Consulta 5 de abril de 2018].

Ballestín González, B. 2015. "De 'su cultura es muy fuerte' a 'no se adapta a la escuela': alumnado de origen inmigrante, evaluación y efecto Pigmalión en primaria". Revista de la Asociación de Sociología de la Educación 8(3): 361-379.

Bernstein, B. 1971. Class, codes and control, Volume 1. London: Routledge.

Blanchard, M. 2009. "El alumnado inmigrante ¿tiene un rendimiento inferior al alumnado autóctono?". Práxis Educacional 5(6): 129-152.

Breen, R. y J. O. Jonsson. 2005. "Inequality of Opportunity in comparative perspective". Annual Review of Sociology 31: 223-243. http://dx.doi.org/10.1146/annurev. soc. 31.041304 .122232 .

Bullejos, J. 2002. "Algunas reflexiones sobre el rendimiento escolar de los estudiantes áraboparlantes en Ceuta y su comparación con los marroquíes de la misma len- que midan simultáneamente ambas variables y que salven las diversas limitaciones y críticas apuntadas.

\section{Agradecimientos}

Los autores agradecen el apoyo en las labores de búsqueda bibliográfica a Laura Gómez Pozo y Ana Lunar Navarro, alumnas colaboradoras en el Departamento de Ciencias Sociales y Humanidades de la Universidad de Córdoba. gua que estudian en los centros españoles de Marruecos". Pp. 213-220 en Inmigración, interculturalidad y convivencia, coordinado por F. Herrera. Ceuta: Instituto de Estudios Ceutíes.

Calero, J., A. Choi y S. Waisgrais. 2009. "Determinantes del rendimiento educativo del alumnado de origen nacional e inmigrante en PISA-2006". Cuadernos económicos de ICE 78: 281-310.

Calero, J., A. Choi y S. Waisgrais. 2010. "Determinantes del riesgo de fracaso escolar en España: una aproximación a través de un análisis logístico multinivel aplicado a PISA-2006". Revista de Educación n. extraordinario: 225-256.

Calero, M. D., A. Fernández-Parra, S. López-Rubio, R. Carles, S. Mata, M. C. Vives, E. Navarro y J. Márquez. 2013. "Variables involved in personal, social and school adjustment in a sample of preschool-aged children from different cultural backgrounds". European journal of psychology of education 28(1): 133-155.

Cano, E. y Fernández, M. 2015. "La visión de los estudiantes de origen extranjero sobre factores y competencias clave para el acceso a la universidad". Pedagogía social: revista interuniversitaria 26: 263-284.

Carabaña, J. 2008. Las diferencias entre regiones y países en las pruebas PISA. Documentos del Colegio Libre de Eméritos. https://colegiodeemeritos.es/wp-content/ uploads/2020/05/pisa_carabana_vf.pdf [Consulta 1 de Febrero de 2016].

Carabaña, J. 2011. "Why Do the Results of Immigrant Students Depend So Much on Their Country of Origin and so Little on Their Country of Destination?". Pp. 207-221 en Pisa Under Examination, editado por M. Pereyra, H. G. Kotthoff y R. Cowen. Rotterdam: Sense Publishers.

Carabaña, J. 2016. "El Informe Coleman, 50 años después". Revista de la Asociación de Sociología de la Educación 9(1): 9-21.

Cebolla-Boado, H. 2008. “¿Están los estudiantes de origen inmigrante en desventaja?: diferencias internacionales e interregionales en España". Panorama social 8: 97-111.

Cebolla-Boado, H. y L. Garrido. 2008. "Sobre la desventaja educativa de los inmigrantes". Indice: Revista de Estadística y Sociedad 30: 21-23.

Cebolla-Boado, H. y A. González Ferrer. 2008. La inmigración en España (2001-2007). De la gestión de los flujos a la integración de los inmigrantes. Madrid: Centro de Estudios Políticos y Constitucionales.

Colectivo IOÉ 1996. La educación intercultural a prueba. Hijos de inmigrantes marroquíes en la escuela. Madrid: CIDE-MEC. 
Colectivo IOÉ 2003. "Alumnos y alumnas de origen extranjero. Distribución y trayectorias diferenciadas". Cuadernos de Pedagogía 326: 63-68.

Cummins, J. 1981. "The role of primary language development in promoting educational succes for language minority students". Pp. 3-50 en Schooling and Language Minority Students: A Theorethycal Framework, editado por California State Department of Education. Los Angeles: Evaluation, Dissemination and Assessment Center-California State University.

Cummins, J. 1984. "Language proficiency and academic achievement revisited: A response". Pp. 71-76 en Language Proficiency and Academic Achievement, editado por C Rivera. Washington DC: Multilingual Matters.

Cummins, J. 2002. Lenguaje, poder y pedagogía: niños y niñas bilingües entre dos fuegos. Madrid: Ediciones Morata.

Cummins, J. 2008. "BICS and CALP: Empirical and theoretical status of the distinction". Pp. 71-83 en Encyclopedia of language and education, Volume 2, editado por B. Street y N. H. Hornberger. New York: Springer.

Defensor Del Pueblo. 2003. Escolarización del alumnado de origen inmigrante en España: análisis descriptivo y estudio empírico. Madrid: Defensor del Pueblo. http:// www.defensordelpueblo.es/es/Documentacion/Publicaciones/monografico/contenido 1261584551261. html [Consulta 20 de enero de 2016]

Díaz-Aguado, M. J., A. Baraja y P. Royo. 1996. "Estudio sobre la integración escolar de los alumnos extranjeros y el aprendizaje del castellano como segunda lengua". Pp. 101-154 en Escuela y tolerancia, editado por M. J. Díaz-Aguado. Madrid: Pirámide.

Edelsky, C., S. Hudelson, B. Altwerger, B. Flores, F. Barkin y K. Jilbert. 1983. "Semilingualism and language deficit". Applied Linguistics 4(1): 1-22. https://doi.org/10.1093/ applin/4.1.1.

Etxeberria, F. 2005. "Lenguas inmigrantes en la escuela en España”. Cuadernos Interculturales, 3(5): 9-30.

Etxeberria, F., J. Garmendia, H. Murua y E. Arrieta. 2019. "Alumnado inmigrante y competencia plurilingüe en la escuela inclusiva". Consulta 5 de abril de 2018. https:// edoc.hu-berlin.de/handle/18452/21343

Foster, P., y M. Hammersley. 1998. "A review of reviews: structure and function in reviews of educational research". British Educational Research Journal 24(5): 609-628.

Franzé Mudano, A. 2008. "Diversidad cultural en la escuela. Algunas contribuciones antropológicas". Revista de educación 345: 111-132.

Fullana, J., M. Vilá y X. Besalú. 2003. Alumnes d'origen africà a l'escola. Girona: CCG Edicions.

García Castaño, F.J. y A. Olmos Alcaraz. 2012. Segregaciones y la construcción de la diferencia en la escuela. Madrid: Trotta.

García Castaño, F.J., M. Rubio Gómez y O. Bouachra. 2015. "Immigrant Students at School in Spain: Constructing a Subject of Study". Dve domovini/Two Homelands 41: 35-47.

Genesee, F. 1984. On Cummins' theoretical framework. Pp. 20-27 en Language Proficiency and Academic Achievement, editado por C. Rivera. Washington DC: Multilingual Matters.

González, X., Á. Huguet y S. Chireac. 2013. "Diversidad lingüística y conocimiento de catalán/castellano. Un estudio empírico con alumnado inmigrante en Cataluña". Teoría de la Educación 25(1): 191-213.

González-Betancor, S. y A. López-Puig. 2008. "Evolución del resultado educativo en España según PISA 2003 y 2006". Investigaciones de Economía de la Educación 3: $145-152$
Gough, D., Thomas, J. y S. Oliver. 2012. "Clarifying differences between review designs and methods". Systematic $R e-$ views 1(1): 28. https://doi.org/10.1186/2046-4053-1-28

Hakuta, K. 1986. Mirror of Language: The Debate on Bilingualism. New York: Basic Books.

Heath, A., C. Rothon y E. Kilpi, 2008. "The Second Generation in Western Europe: Education, Unemployment, and Occupational Attainment". Annual Review of Sociology 34: 211-235. https://doi.org/10.1146/annurev. soc. 34.040507 .134728

Huguet, A. 2008. "Interdependencia lingüística y práctica educativa. Un estudio con escolares de origen inmigrante". Infancia y Aprendizaje 31(3): 283-301. https://doi. org/10.1174/021037008785702901

Huguet, Á. y J. L. Navarro. 2006. "Inmigración y resultados escolares: loque dicelainvestigación”. Culturay Educación 18(2): 117-126. https://doi.org/10.1174/113564006777973879

Huquet, A., J. L. Navarro y J. Janés. 2007. "La adquisición del castellano por los escolares inmigrantes. El papel del tiempo de estancia y la lengua familiar". Anuario de Psicología 38(3): 357-375

Huguet, Á. y J. Janés. 2013. "Escuela e inmigración: una presentación de estudios sobre el desarrollo de habilidades lingüísticas y procesos de interdependencia entre lenguas". Anales de psicología 29(2): 393-402. http:// dx.doi.org/10.6018/analesps.29.2.130572

Kao, G. y M. Tienda. 2005. "Optimism and achievement: The educational performance of immigrant youth". Pp. 331343 en The new immigration: An interdisciplinary reader, editado por M. Suarez-Orozco, C. Suarez-Orozco y D. Baolian Qin. New York: Routledge.

Levels, M., J. Dronkers y G. Kraaykamp. 2008. "Immigrant Children's Educational Achievement in Western Countries: Origin, Destination, and Community Effects on Mathematical Performance". American Sociological Review 73: 835-853. https://doi. org/10.1177/000312240807300507

MacSwan, J. y K. Rolstad. 2003. "Linguistic diversity, schooling and social class: Rethinking our conception of language proficiency in language minority education". Pp. 329340 en Sociolinguistics: The essential readings, editado por C. B. Paulston y R. Tucker. Oxford: Blackwell.

MacSwan, J. y K. Rolstad. 2010. "The role of language in theories of academic failure for linguistic minorities". Pp. 173-195 en International perspectives on bilingual education: Policy, practice, and controversy, editado por J. Petrovic. Charlotte, NC: Information Age.

Martin-Jones, M. y S. Romaine. 1986. "Semilingualism: A halfbaked theory of communicative competence". Applied Linguistics 7(1): 26-38.

Medvedeva, M. y A. Portes. 2016. "Immigrant Bilingualism in Spain: An Asset or a Liability?". International Migration Review 51 (3): 632-666. https://doi.org/10.1111/imre.12243

Mesa, M. C. y S. Sánchez y M. M. Vázquez. 1996. Educación y situaciones bilingües en contextos multiculturales. Estudio de un caso: Melilla. Granada: Laboratorio de Estudios Interculturales- CIDE.

Ministerio de Educación. 2018. "Enseñanzas no universitarias". Madrid: Ministerio de Educación. https://www.educacionyfp.gob.es/servicios-al-ciudadano/estadisticas/nouniversitaria.html [Consulta 29 de noviembre de 2019].

Navarro, J. L. y A. Huguet. 2005. El conocimiento de la lengua castellana en alumnado emigrante de $1^{\circ}$ de ESO. Un estudio empírico. Madrid: CIDE.

Navarro, J. L. y A. Huguet. 2010. "Conocimiento del catalán y castellano por parte del alumnado inmigrante". Revista de Educación 352: 245-265 
Navarro, J. L., A. Huguet, C. Sansó y S. M. Chireac. 2012. "Acerca de la competencia lingüística del alumnado de origen inmigrante en la ESO en Cataluña. El papel del tiempo de estancia y la lengua familiar". Anales de Psicología 28(2): 457-464. https://doi.org/10.6018/ analesps.28.2.148841

Navarro, J.L., A. Huguet y C. Sansó. 2014. "Competencias lingüísticas y alumnado inmigrante en Cataluña. El caso del colectivo de origen hispanohablante". Educación XX1 17(2): 361-382.

OECD. 2010. PISA 2009 Results: What Students Know and Can Do-Student Performance in Reading, Mathematics and Science (Volume I). https://www.oecd.org/pisa/pisaproducts/48852548.pdf [Consulta 24 de enero de 2016].

OECD. 2011. "How Are School Systems Adapting to Increasing Numbers of Immigrant Students?". Pisa in Focus, $\mathrm{n}^{\circ} 11$. Paris: OECD Publishing. http://www.oecd-ilibrary.org/ education/how-are-school-systems-adapting-to-increasing-numbers-of-immigrant-students $5 \mathrm{k} 9 \mathrm{~h} 362 \mathrm{hs} 646$ en [Consulta 24 de enero de 2016].

OECD. 2016. PISA 2015 Results: Excellence and Equity in Education, Volume I. Paris: OECD Publishing. http:/l dx.doi.org/10.1787/9789264266490-en [Consulta 12 de noviembre de 2019].

Oller, J. y I. Vila. 2008. "El conocimiento del catalán y el castellano del alumnado de origen extranjero, tiempo de estancia en Cataluña y lengua inicial al finalizar la enseñanza primaria". Segundas lenguas e inmigración en red $\mathrm{I}(1)$ : $10-24$.

Pàmies, J. 2006. "Dinámicas escolares y comunitarias de los hijos e hijas de familias inmigradas marroquíes de la Yebala en la periferia de Barcelona". Tesis doctoral, Departament Antropología Social i Cultural, Universitat Autònoma de Barcelona, Barcelona, España.

Portes, A., R. Aparicio Gómez y W. Haller. 2016. Spanish Legacies: The Coming of Age of the Second Generation. Los Angeles: University of California Press.

Portes, A. y A. Rivas. 2011. "The adaptation of migrant children". The future of children 21(1): 219-246.

Rahona, M. y S. Morales. 2013. Educación e inmigración en España: desafíos y oportunidades. Madrid: Organización de Estados Iberoamericanos.

Reher, D. y M. Requena. 2009. "The National Immigrant Survey of Spain: A new data source for migration studies in Europe". Demographic Research 20: 253-278. https:// doi.org/10.4054/DemRes.2009.20.12

Rindermann, H. y J. Thompson. 2016. "The cognitive competences of immigrant and native students across the world: An analysis of gaps, possible causes and impact". Journal of biosocial science 48: 66-93. https:// doi.org/ 10.1017/S0021932014000480

Rivera, C. 1984. Language proficiency and academic achievement. Clevedon: Multilingual Matters.

Rubio Gómez, M. 2013. Construyendo diferencias desde las retóricas de la igualdad. Granada: Universidad de Granada.

Salinas, J. y D. Santín. 2012. "Selección escolar y efectos de la inmigración sobre los resultados académicos españoles en PISA 2006". Revista de Educación 358: 382-405.
Sánchez-Meca, J. y J. Botella. 2010. Revisiones sistemáticas y meta-análisis: herramientas para la práctica profesional. Papeles del Psicólogo, 31(1). http://www.redalyc. org/articulo.oa?id=77812441002

Schnell, P. y D. Azzolini. 2015. "The academic achievements of immigrant youths in new destination countries: Evidence from southern Europe". Migration Studies 3(2): 217-240. http://dx.doi.org/10.1093/migration/mnu040

Serra i Salamé, C. y J. Miquel Palaudàrias. 2010. Continuar o abandonar. L'alumnat estranger a l'educació secundària. Barcelona: Fundació Jaume Bofill.

Siguan, M. 1998. La escuela y los inmigrantes. Barcelona: Paidós.

Stanat, P. y G. Christensen. 2006. Where immigrant students succeed: A comparative review of performance and engagement in PISA 2003. París: OECD.

Stevens, P. A. 2007. "Researching race/ethnicity and educational inequality in English Secondary Schools: A critical review of the research literature between 1980 and 2005". Review of Educational Research 77(2): 147185. https://doi.org/10.3102/003465430301671

Stevens, P. A., N. Clycq, C. Timmerman y M. Van Houtte 2011. "Researching race/ethnicity and educational inequality in the Netherlands: a critical review of the research literature between 1980 and 2008". British Educational Research Journal 37 (1): 5-43. https://doi. org/10.1080/01411920903342053

Stolzenberg, R. y M. Tienda. 1997. "English Proficiency, Education, and the Conditional Economic Assimilation of Hispanic and Asian Origin Men". Social Science Research 25: 26-51.

Terrén Lalana, E. 2008. "La integración educativa de los hijos de familias inmigradas". Documento de trabajo para el VI Informe sobre exclusión y desarrollo social en España 2008. https://www.caritas.es/producto/vi-informeexclusion-desarrollo-social-espana-2008/

Troike, R.C. 1984. "SCALP: Social and cultural aspects of language proficiency". Pp. 55-70 en Language Proficiency and Academic Achievement, editado por C. Rivera. Washington DC: Multilingual Matters.

Vila, I. 2008. "Lengua familiar y conocimiento de la lengua escolar en Cataluña al finalizar la Educación Infantil". Revista de Educación 346: 401-424.

Vila, I. 2011. "La evaluación del alumnado extranjero y su conocimiento lingüístico". Pp. 71-86 en Evaluar la competencia lingüística del alumnado inmigrante, coordinado por I. Ballano. Bilbao: Deusto Digital.

Waters, M. C., A. Heath, V. Tran y V. Boliver. 2013. "Second Generation Attainment and Inequality: Primary and Secondary Effects on Educational Outcomes in Britain and the United States". Pp. 120-159 en The Children of Immigrants at School, editado por R. Alba y J. Holdaway. New York: New York University Press.

Wiley, T. G. 1996. Literacy and language diversity in the United States. Washington, DC: Center for Applied Linguistics and Delta Systems.

Zinovyeva, N., F. Felgueroso y P. Vázquez. 2013. "Immigration and student achievement in Spain: evidence from PISA". SERIES 5(1): 25-60.

ALBERTO ÁLVAREZ-SOTOMAYOR es doctor en Sociología por la Universidad de Granada. Actualmente es profesor ayudante doctor en el Departamento de Ciencias Sociales y Humanidades de la Universidad de Córdoba. Sus trabajos se desarrollan principalmente en dos áreas: la sociología de la educación y la sociología de las migraciones internacionales. Entre sus intereses investigadores destaca el análisis de los procesos de integración de la población inmigrante en España y, en especial, el estudio del rendimiento académico de los hijos de inmigrantes.

GLORIA MARTÍNEZ-COUSINOU es doctora en Ciencias Políticas por la UNED y premio extraordinario de doctorado por esta universidad. Profesora e investigadora en el Departamento de Estudios Internacionales de la Universidad Loyola Andalucía. Sus trabajos se enmarcan en la línea de investigación sobre gobierno, políticas públicas y políticas sociales. 